Draft Version MAY 24, 2021

Preprint typeset using LTEX style emulateapj v. 12/16/11

\title{
LOW-LUMINOSITY TYPE IIP SUPERNOVA 2016BKV WITH EARLY-PHASE CIRCUMSTELLAR INTERACTION
}

\author{
Tatsuya NaKaoka ${ }^{1}$, Koj S. Kawabata ${ }^{1,2}$, Keitchi Maeda ${ }^{3}$, Masaomi Tanaka ${ }^{4}$, Masayuki Yamanaka ${ }^{2,5}$, Takashi J. Moriya ${ }^{4}$, Nozomu Tominaga ${ }^{5}$, \\ Tomoki Morokuma ${ }^{6}$, Katsutoshi Takaki ${ }^{1}$, Mino Kawabata ${ }^{1}$, Naoki Kawahara ${ }^{1}$, Ryosuke Itoh ${ }^{1,7}$, Kensei Shiki ${ }^{1}$, Hiroki Mori ${ }^{1}$, Jun Hirochi ${ }^{1}$, \\ Taisei Abe ${ }^{1}$, Makoto Uemura ${ }^{1,2}$, Michitoshi Yoshida ${ }^{2,8}$, Hiroshi Akitaya ${ }^{2,9}$, Yuki Moritani ${ }^{2,10}$, Issei Ueno ${ }^{1}$, Takeshi Urano ${ }^{1}$, Mizuki Isogai ${ }^{4,11}$, \\ Hidekazu Hanayama ${ }^{12}$, and Takahiro Nagayama ${ }^{13,14}$, \\ Draft version May 24, 2021
}

\begin{abstract}
We present optical and near-infrared observations of a low-luminosity Type IIP supernova (SN) 2016bkv from the initial rising phase to the plateau phase. Our observations show that the end of the plateau is extended to $\gtrsim 140$ days since the explosion, indicating that this SN takes one of the longest time to finish the plateau phase. among Type IIP SNe (SNe IIP), including low-luminosity (LL) SNe IIP. The line velocities of various ions at the middle of the plateau phase are as low as $1,000-1,500 \mathrm{~km} \mathrm{~s}^{-1}$, which is the lowest even among LL SNe IIP. These measurements imply that the ejecta mass in SN 2016bkv is larger than that of the well-studied LL IIP SN 2003Z. In the early phase, SN 2016bkv shows a strong bump in the light curve. In addition, the optical spectra in this bump phase exhibit a blue continuum accompanied with a narrow $\mathrm{H} \alpha$ emission line. These features indicate an interaction between the SN ejecta and the circumstellar matter (CSM) as in $\mathrm{SNe}$ IIn. Assuming the ejecta-CSM interaction scenario, the mass loss rate is estimated to be $\sim 1.7 \times 10^{-2} M_{\odot} \mathrm{yr}^{-1}$ within a few years before the SN explosion. This is comparable to or even larger than the largest mass loss rate observed for the Galactic red supergiants $\left(\sim 10^{-3} M_{\odot} \mathrm{yr}^{-1}\right.$ for VY CMa). We suggest that the progenitor star of SN 2016bkv experienced a violent mass loss just before the SN explosion.
\end{abstract}

Keywords: supernovae: general - supernovae: individual (SN 2016bkv)

\section{INTRODUCTION}

Type IIP supernovae (SNe IIP) are characterized by hydrogen features in their early-phase spectra and the 'plateau' in their optical light curves lasting for $\sim 100$ days (Filippenko 1997). SNe IIP represent the most common class of SNe and occupy $\sim 70 \%$ of core-collapse SNe within $60 \mathrm{Mpc}$ (Li et al. 2011; Graur et al. 2017; Shivvers et al. 2017). The

\footnotetext{
${ }^{1}$ Department of Physical Science, Hiroshima University, Kagamiyama 1-3-1, Higashi-Hiroshima 739-8526, Japan; nakaoka@ astro.hiroshimau.ac.jp

${ }^{2}$ Hiroshima Astrophysical Science Center, Hiroshima University, Higashi-Hiroshima, Hiroshima 739-8526, Japan

${ }^{3}$ Department of Astronomy, Kyoto University, Kitashirakawa-Oiwakecho, Sakyo-ku, Kyoto 606-8502, Japan

${ }^{4}$ National Astronomical Observatory of Japan, 2-21-1 Osawa, Mitaka, Tokyo 181-8588, Japan

${ }^{5}$ Department of Physics, Faculty of Science and Engineering, Konan University, Kobe, Hyogo 658-8501, Japan

${ }^{6}$ Institute of Astronomy, Graduate School of Science, The University of Tokyo, 2-21-1 Osawa, Mitaka, Tokyo 181-0015, Japan

${ }^{7}$ School of Science, Tokyo Institute of Technology, 2-12-1 Ohokayama, Meguro-ku, Tokyo 152-8551, Japan

${ }^{8}$ Subaru Telescope, National Astronomical Observatory of Japan, 650 North A'ohoku Place, Hilo, HI 96720, USA

${ }^{9}$ Graduate School of Science and Engineering, Saitama University,255 Shimo-Okubo, Sakura-ku, Saitama, 338-8570, Japan

${ }^{10}$ Kavli Institute for the Physics and Mathematics of the Universe (WPI), The University of Tokyo Institutes for Advanced Study, The University of Tokyo, 5-1-5 Kashiwanoha, Kashiwa, Chiba 277-8583, Japan

${ }^{11}$ Graduate School of Science and Engineering, Kagoshima University, 1-21-35 Korimoto, Kagoshima 890-0065, Japan 13 Gunma Astronomical Observatory, Takayama, Gunma 377-0702, Japan

12 Okayama Astrophysical Observatory, National Astronomical Observatory of Japan, National Institutes of Natural Sciences, 3037-5 Honjo, Kamogata, Asakuchi, Okayama 719-0232, Japan

${ }^{13}$ Department of Astrophysics, Nagoya University, Furo-cho, Chikusaku, Nagoya, Aichi 464-8602, Japan

${ }^{14}$ Koyama Astronomical Observatory, Kyoto Sangyo University, Motoyama, Kamigamo, Kita-Ku, Kyoto, Kyoto, 603-8555, Japan
}

observational properties of SNe IIP show various diversities, for example, in the durations of the plateau and the shapes of the initial light curves (Anderson et al. 2014; Faran et al. 2014; Sanders et al. 2015; Peicha \& Prieto 2015; Valenti et al. 2016). So far, clear early-phase bump features have been found in several SNe II (e.g., Khazov et al. 2016). The early bump emission can be explained by the interaction between the SN ejecta and the circumstellar medium (CSM), which is originated from a stellar wind of the progenitor star (Moriya et al. 2017; Morozova et al. 2017; Yaron et al. 2017; Dessart et al. 2017). These diversities may well be related to different natures of their progenitors.

The absolute magnitudes in the plateau phase also show a large variety, ranging from $\sim-14$ to $\sim-17$ mag (Anderson et al. 2014). Note that this range is derived including some SNe IIL, which tend to show the brightest luminosities in the sample (i.e., -17 mag; Bose et al. 2015). In this paper, we refer to typical SNe IIP with the absolute plateau magnitudes of $\sim-16$ or -17 mag as "normal SNe IIP'. In the past $\sim 20$ years, a subclass of faint SNe IIP has been recognized, called low-luminosity SNe IIP (LL SNe IIP; Pastorello et al. 2004). A typical luminosity of LL SNe IIP is lower than those of normal SNe IIP by 2-3 mag. Typical velocities of the absorption lines are also lower than those of normal SNe IIP by a factor of 3-4 (Pastorello et al. 2004). The origin of these LL SNe IIP is still under debate: They may be either originated from weak explosions of the less massive stars $\left(\sim 9 M_{\odot}\right)$ or failed explosions of the massive stars $\left(\sim 25 M_{\odot}\right)$ (Turatto et al. 1998; Chugai \& Utrobin 2000; Kitaura et al. 2006). To date, several LL SNe IIP have been well studied (e.g., Turatto et al. 1998; Pastorello et al. 2004; Spiro et al. 2014; Lisakov et al. 2017, 2018). However, the sample covering both the initial rising phase and the tail after the plateau phase is still small. Especially, the observations in near-infrared (NIR) wavelengths are still rare for LL SNe IIP. In this paper, we present our observations of a LL SN 
IIP 2016bkv. SN 2016bkv was discovered by Koichi Itagaki on 2016 Mar 21.7 (UT) (Itagaki 2016). At two days after the discovery, this SN was classified as an SN II, by the spectral similarity to SN IIn 1998S (Hosseinzadeh et al. 2016). Interestingly, this SN shows an unusually strong initial bump in the light curves. The host galaxy, NGC 3184, is a face-on spiral galaxy and known to be an active $\mathrm{SN}$ factory in the last 100 years (e.g., SNe 1921B, 1921C, 1937F and 1999gi; Leonard et al.2002b), The distance to the host galaxy is assumed to be $12.3 \pm 2.2 \mathrm{Mpc}$, adopting the mean redshiftindependent distance reported in the literatures (via NED 15 ). The explosion date is assumed to be 2016 Mar 20.4 \pm 1.3 , as constrained by the last non-detection date (Mar 19.1) by Katzman Automatic Imaging Telescope (KAIT). Throughout the paper, the observation epoch is given with respect to the estimated explosion date. Hosseinzadeh et al. (2018) presented optical light curves and spectra of SN 2016bkv, and discussed the nature of the explosion and progenitor using models of nebular spectra. Our paper provides complementary data including optical and NIR light curves with a longer plateau coverage.

This paper is organized as follows. In $\S 2$, we describe our observations and data reduction. In $\S 3$ and 4 , we show photometric and spectroscopic properties of SN 2016bkv, respectively. We discuss the nature of this object in $\$ 5$, and finally, we give conclusions in $\S 6$

\section{OBSERVATIONS AND DATA REDUCTION}

The optical imaging data were obtained using the Hiroshima One-shot Wide-field Polarimeter (HOWPol; Kawabata et al. 2008) and the Hiroshima Optical and NearInfraRed Camera (HONIR; Akitaya et al. 2014). These instruments were installed into the 1.5-m Kanata telescope at the Higashi-Hiroshima Observatory, Hiroshima University. We obtained $B V R I$-band data using HOWPol in 10 nights from 2016 Apr 5.3 until Oct 9.4, and also obtained BVRIband data using HONIR in 14 nights from Mar 24.3 until Aug 9.1. For the photometric measurements, we adopted the Point Spread Function (PSF) photometry task in the DAOPHOT package (Stetson 1987) equipped with the IRAF (Tody 1986, 1993). For the calibration of optical photometry, we used the magnitudes of a nearby comparison star given in Leonard et al. (2002b) (see Figure 1). The derived optical magnitudes are summarized in Table 1. Figure 2 shows the light curve in each band. All magnitudes are given in the Vega magnitudes throughout the paper.

In the late phase ( $\gtrsim 150$ days), background emission from the host galaxy may contribute to the total measured flux. To constrain possible contamination, we use a pre-explosion image taken by the Hubble Space Telescope (HST) on 2001 Jan 24. We found a probable stellar cluster at $m_{\mathrm{F} 555 \mathrm{~W}}=19-20$ mag within the typical PSF radius of our observations $\left(\sim 2^{\prime \prime}\right)$ around the SN. Since the brightness in the plateau phase is much brighter than that of this source, our magnitudes up to 150 days are reliable within a systematic error of $\simeq 0.1 \mathrm{mag}$ in optical bands. On the other hand, the magnitudes in the last night (at 203 days) are close to the background source, and thus we treat this brightness as upper limits of SN fluxes. Throughout the paper, we focus on the phases before 150 days and our main discussion is not affected by the presence of the cluster-like component.

The $J H K_{s}$-band imaging data were obtained using HONIR

15 http://ned.ipac.caltech.edu/

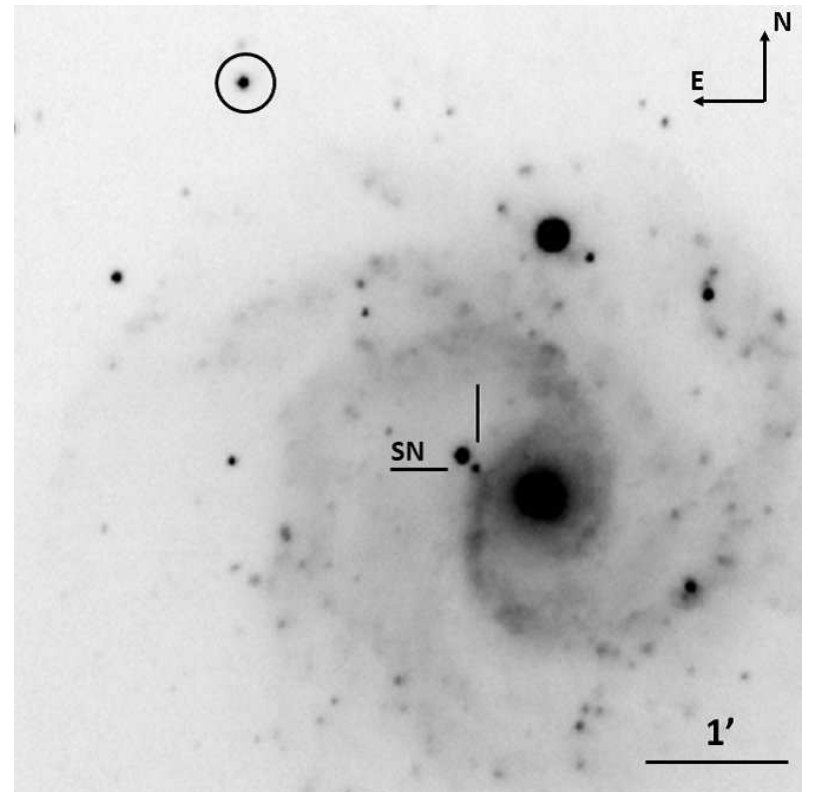

Figure 1. $R$-band image of SN 2016bkv in NGC 3184 taken using HOWPol. The location of SN and comparison star (Leonard et al. 2002b) are marked.

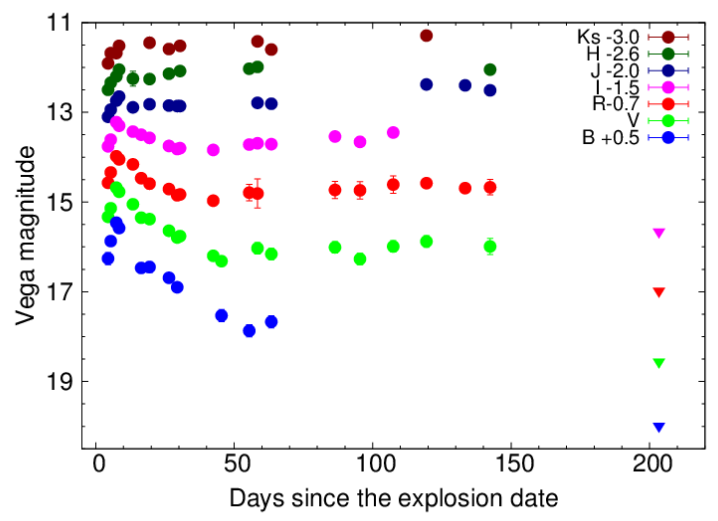

Figure 2. Optical to NIR light curves of SN 2016bkv. The Galactic interstellar extinction (Schlafly \& Finkbeiner 2011) has been corrected for. The host-galactic extinction is estimated to be negligible (see $\$ 3$ ) and thus no correction has been made.

in 14 nights from Mar 24.3 until Aug 9.1. We took the images using a dithering mode to accurately subtract the bright foreground sky. We reduced the data according to the standard manner for the NIR data using the PSF photometry method in $I R A F$. The magnitude calibrations were performed using the magnitudes of nearby comparison stars given in the 2MASS catalog (Persson et al. 1998). The derived $J H K_{s}$-band magnitudes and their light curves are given in Table 2 and Figure 2 respectively.

We performed optical spectroscopic observations using HOWPol in 17 nights from Mar 24.3 until Jun 26.3. We used a grism with a spectral resolution of $R \sim 400$ and a spectral coverage of 4500-9000 $\AA$. We observed spectroscopic standard stars in the same nights for the flux calibrations. For the wavelength calibration, we used the sky emission lines taken in the object frames. The strong atmospheric absorption bands around $6900 \AA$ and $7600 \AA$ have been removed using the smooth spectra of the hot standard stars. The obtained 
Table 1

Photometric observations of SN 2016bkv in optical bands.

\begin{tabular}{lllllll}
\hline \hline MJD & $\begin{array}{l}\text { Epoch } \\
(\text { day })\end{array}$ & $\begin{array}{l}\text { B } \\
(\mathrm{mag})\end{array}$ & $\begin{array}{l}\mathrm{V} \\
(\mathrm{mag})\end{array}$ & $\begin{array}{l}\mathrm{R} \\
(\mathrm{mag})\end{array}$ & $\begin{array}{l}\mathrm{I} \\
(\mathrm{mag})\end{array}$ & Instrument \\
\hline 57471.7 & 4.4 & $15.76(0.13)$ & $15.33(0.08)$ & $15.27(0.04)$ & $15.26(0.04)$ & HONIR \\
57472.7 & 5.4 & $15.37(0.07)$ & $15.14(0.06)$ & $15.04(0.04)$ & $15.11(0.04)$ & HONIR \\
57474.7 & 7.4 & $14.96(0.05)$ & $14.68(0.04)$ & $14.68(0.04)$ & $14.72(0.04)$ & HONIR \\
57475.7 & 8.4 & $15.08(0.04)$ & $14.77(0.05)$ & $14.75(0.04)$ & $14.80(0.05)$ & HONIR \\
57480.7 & 13.4 & - & $15.05(0.05)$ & $14.86(0.04)$ & $14.93(0.08)$ & HONIR \\
57483.6 & 16.3 & $15.97(0.04)$ & $15.35(0.05)$ & $15.17(0.04)$ & $15.0(0.08)$ & HOWPol \\
57486.6 & 19.3 & $15.95(0.11)$ & $15.38(0.05)$ & $15.29(0.11)$ & $15.07(0.05)$ & HONIR \\
57493.6 & 26.3 & $16.19(0.04)$ & $15.64(0.05)$ & $15.41(0.05)$ & $15.25(0.05)$ & HONIR \\
57496.7 & 29.4 & $16.40(0.08)$ & $15.79(0.07)$ & $15.55(0.07)$ & $15.32(0.04)$ & HONIR \\
57497.7 & 30.4 & - & $15.76(0.06)$ & $15.53(0.04)$ & $15.30(0.04)$ & HONIR \\
57509.7 & 42.4 & - & $16.2(0.06)$ & $15.67(0.04)$ & $15.34(0.04)$ & HOWPol \\
57512.6 & 45.3 & $17.03(0.13)$ & $16.32(0.06)$ & - & - & HOWPol \\
57522.6 & 55.3 & $17.37(0.13)$ & - & $15.49(0.18)$ & $15.22(0.06)$ & HONIR \\
57525.6 & 58.3 & - & $16.03(0.12)$ & $15.51(0.32)$ & $15.19(0.05)$ & HONIR \\
57530.6 & 63.3 & $17.17(0.13)$ & $16.16(0.12)$ & - & $15.21(0.08)$ & HONIR \\
57553.6 & 86.3 & - & $16.01(0.12)$ & $15.43(0.19)$ & $15.04(0.08)$ & HOWPol \\
57562.5 & 95.2 & - & $16.27(0.12)$ & $15.44(0.19)$ & $15.16(0.08)$ & HOWPol \\
57574.5 & 107.2 & - & $15.99(0.12)$ & $15.31(0.19)$ & $14.95(0.08)$ & HOWPol \\
57586.5 & 119.2 & - & $15.88(0.13)$ & $15.28(0.04)$ & - & HONIR \\
57600.5 & 133.2 & - & - & $15.39(0.07)$ & - & HONIR \\
57609.5 & 142.2 & - & $15.99(0.18)$ & $15.37(0.17)$ & - & HONIR \\
57670.8 & 203.5 & $19.49(0.13)$ & $18.56(0.18)$ & $17.68(0.17)$ & $17.16(0.08)$ & HOWPol \\
& & & & & &
\end{tabular}

Table 2

Photometric observations of SN 2016bkv in NIR bands.

\begin{tabular}{lllll}
\hline \hline MJD & $\begin{array}{l}\text { Epoch } \\
(\text { day })\end{array}$ & $\begin{array}{l}\text { J } \\
(\mathrm{mag})\end{array}$ & $\begin{array}{l}\mathrm{H} \\
(\mathrm{mag})\end{array}$ & $\begin{array}{l}\text { Ks } \\
(\mathrm{mag})\end{array}$ \\
\hline 57471.7 & 4.4 & $15.1(0.02)$ & $15.1(0.03)$ & $14.91(0.03)$ \\
57472.7 & 5.4 & $14.94(0.02)$ & $14.94(0.03)$ & $14.68(0.03)$ \\
57474.7 & 7.4 & $14.74(0.02)$ & $14.8(0.03)$ & $14.68(0.03)$ \\
57475.7 & 8.4 & $14.65(0.02)$ & $14.65(0.03)$ & $14.52(0.04)$ \\
57480.7 & 13.4 & $14.89(0.04)$ & $14.85(0.16)$ & - \\
57486.6 & 19.3 & $14.82(0.02)$ & $14.86(0.05)$ & $14.45(0.07)$ \\
57493.6 & 26.3 & $14.85(0.03)$ & $14.74(0.03)$ & $14.59(0.04)$ \\
57496.7 & 29.4 & $14.86(0.02)$ & - & - \\
57497.7 & 30.4 & $14.86(0.02)$ & $14.68(0.03)$ & $14.52(0.04)$ \\
57522.6 & 55.3 & - & $14.63(0.05)$ & - \\
57525.6 & 58.3 & $14.79(0.05)$ & $14.59(0.02)$ & $14.42(0.06)$ \\
57530.6 & 63.3 & $14.81(0.04)$ & - & $14.6(0.08)$ \\
57586.5 & 119.2 & $14.38(0.04)$ & - & $14.29(0.08)$ \\
57600.5 & 133.2 & $14.4(0.04)$ & - & - \\
57609.5 & 142.2 & $14.51(0.03)$ & $14.65(0.06)$ & - \\
\hline
\end{tabular}

spectra are shown in Figure 3 and the log of our spectroscopic observations is shown in Table 3 .

\section{LIGHT CURVES}

The observed optical and NIR light curves of SN 2016bkv are shown in Figure 2. The Galactic extinction of $E(B-V)=$ 0.0144 (Schlafly \& Finkbeiner 2011) has been corrected for. We also derive an upper limit of the total extinction as $E(B-$ $V) \lesssim 0.015$ by using the equivalent width of $\mathrm{Na}$ I $\mathrm{D}$ absorption line (Poznanski et al. 2012) in our stacked spectrum (EW $\lesssim$ $0.065 \AA$ ). Since the Galactic extinction is close to the upper limit of the total extinction, we consider that the interstellar extinction within the host galaxy is negligibly small ( $E(B-$ $V) \lesssim 0.005)$.

Figure 4 shows the absolute $R$-band light curve of SN 2016bkv, compared with those of other LL SNe IIP and a normal SN IIP 1999em. For the SNe other than SN 2016bkv, we adopted the estimates of the explosion dates from Pastorello et al. (2004), Fraser et al. (2011) and Spiro et al. (2014) and the total extinction corrections used in
Table 3

Spectroscopic observations of SN 2016bkv.

\begin{tabular}{lll}
\hline \hline MJD & $\begin{array}{l}\text { Epoch } \\
\text { (day) }\end{array}$ & $\begin{array}{l}\text { Exposure } \\
(\mathrm{sec})\end{array}$ \\
\hline 57471.5 & 4.2 & 1600 \\
57472.7 & 5.4 & 1400 \\
57474.8 & 7.5 & 900 \\
57475.6 & 8.3 & 1200 \\
57476.7 & 9.4 & 1150 \\
57477.7 & 10.4 & 1200 \\
57483.6 & 16.3 & 1200 \\
57486.5 & 19.2 & 1500 \\
57489.5 & 22.2 & 1500 \\
57493.5 & 26.2 & 1500 \\
57495.6 & 28.3 & 1200 \\
57496.6 & 29.3 & 2400 \\
57512.7 & 45.4 & 1800 \\
57522.6 & 55.3 & 1800 \\
57530.6 & 63.3 & 2700 \\
57553.5 & 86.2 & 2700 \\
57565.5 & 98.2 & 2700 \\
\hline
\end{tabular}

the references. In the mid-plateau phase ( $\sim 80$ days), the $R$ band absolute magnitude of SN $2016 \mathrm{bkv}$ is $M_{R} \sim-15.0 \mathrm{mag}$. This is $\sim 2$ mag fainter than that of the normal SN IIP $1999 \mathrm{em}$. Among LL SNe IIP, SN 2016bkv is $\sim 0.2$ mag brighter than SN $2003 Z$ and $\sim 0.5 \mathrm{mag}$ fainter than that of SN $2002 \mathrm{gd}$. Note that, SN 2016bkv shows a slight brightening toward $\sim 120$ days, reaching $M_{R} \sim-15.1 \mathrm{mag}$.

The light curves of SN 2016bkv have two remarkable characteristics. One is that the end of the plateau phase ( $\gtrsim 140$ days after the explosion) is later than those of other LL SNe IIP for which good data covering the plateau available; $\sim 110$ days in SNe 2003Z and 2009md (Spiro et al. 2014; Fraser et al. 2011). In fact, this epoch is still later than that of SN 2009ib (Takáts et al. 2015), which shows the longest plateau length ( 130 days) among normal SNe IIP.

The other remarkable feature of SN 2016bkv is a bump in the early phase. The light curves show a peak at $\sim 7$ days after the explosion. At 2-7 days, the $\mathrm{SN}$ shows a rapid brightening 


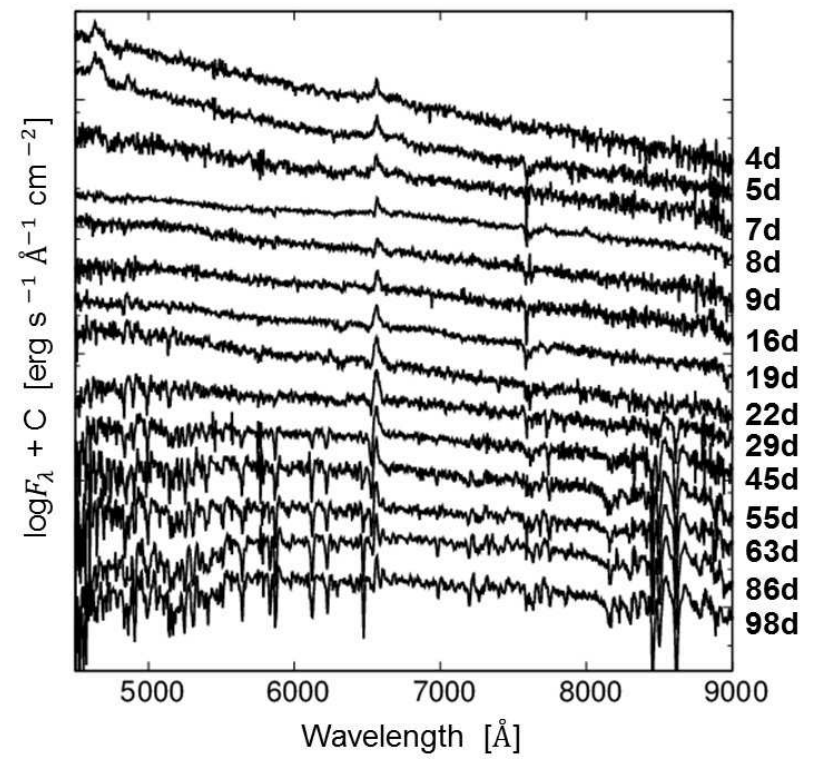

Figure 3. Spectral evolution of SN 2016bkv. The epoch (days after the explosion; see $\S 1$ ) of each spectrum is given in the panel.

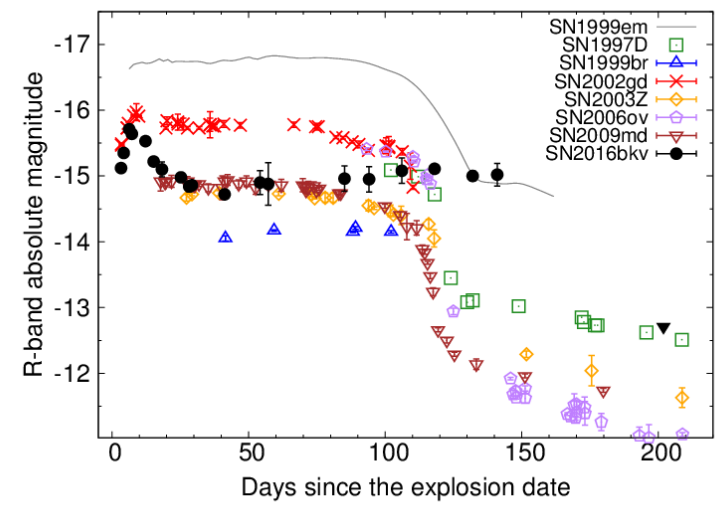

Figure 4. The $R$-band light curve of SN 2016bkv compared with those of other LL IIP SNe 1997D, 1999br (Pastorello et al. (2004), 2002gd, 2003Z, 2006ov (Spiro et al. 2014), 2009md (Fraser et al. 2011) and normal IIP SN 1999em (Leonard et al. 2002a).

by about $0.8 \mathrm{mag}, 0.65 \mathrm{mag}, 0.6 \mathrm{mag}$ and $0.54 \mathrm{mag}$ in the $B$-, $V$-, $R$ - and $I$-band, respectively. Such bumps in the light curves are also seen in the $V$-band light curve of SN $2002 \mathrm{gd}$ (Spiro et al. 2014) and the $B$-band light curve of SN $2005 \mathrm{cs}$ (Pastorello et al. 2009) but the bump in SN 2016bkv is more pronounced. The bump gradually decays (by $\sim 1$ mag through $\sim 30$ days in the $R$-band) and then the $\mathrm{SN}$ seems to enter into the plateau phase.

The decay rates of the bump $\left(s_{1}\right.$, as defined by Anderson et al. 2014) are $\sim 0.043$ and $\sim 0.03 \mathrm{mag} \mathrm{day}^{-1}$ in the $V$-band and $R$-band, respectively. The amplitudes of the bump and their decay rates are larger in shorter wavelengths (Galbany et al. 2016). Figure 5 shows the peak absolute magnitude in the $V$-band $\left(M_{\max }\right)$ and the decay rate of the bump $\left(s_{1}\right)$ of SN $2016 \mathrm{bkv}$ as compared with those of other SNe II (Anderson et al. 2014). The error includes the uncertainty of the distance to the host galaxy and the uncertainty of a transition epoch from the bump into the plateau. From Figure 5 it is clear that SN 2016bkv shows the fastest decay in SNe II having similar peak brightness or LL SNe IIP. We will discuss the features and possible origin of the bump in $\$ 5.4$.

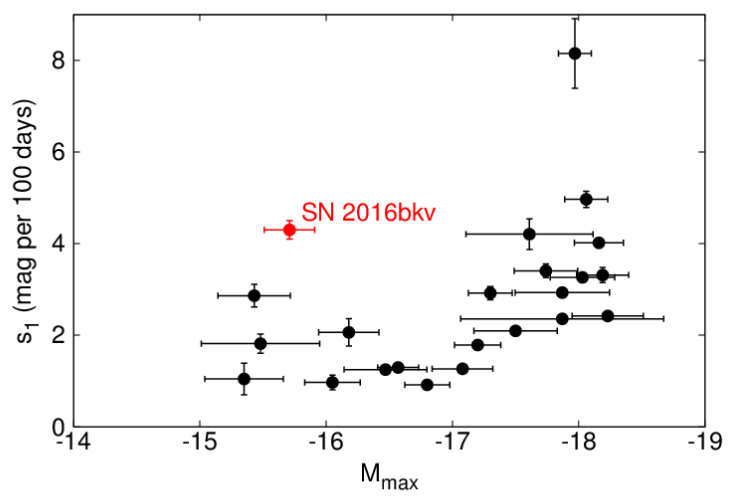

Figure 5. Relation between absolute peak magnitudes in the $V$-band and the decay rates of the initial bumps $\left(s_{1}\right)$ for SN 2016bkv and other SNe II (except for type IIb and IIn). All the data except for SN 2016bkv are taken from Anderson et al. (2014).

\section{SPECTRA}

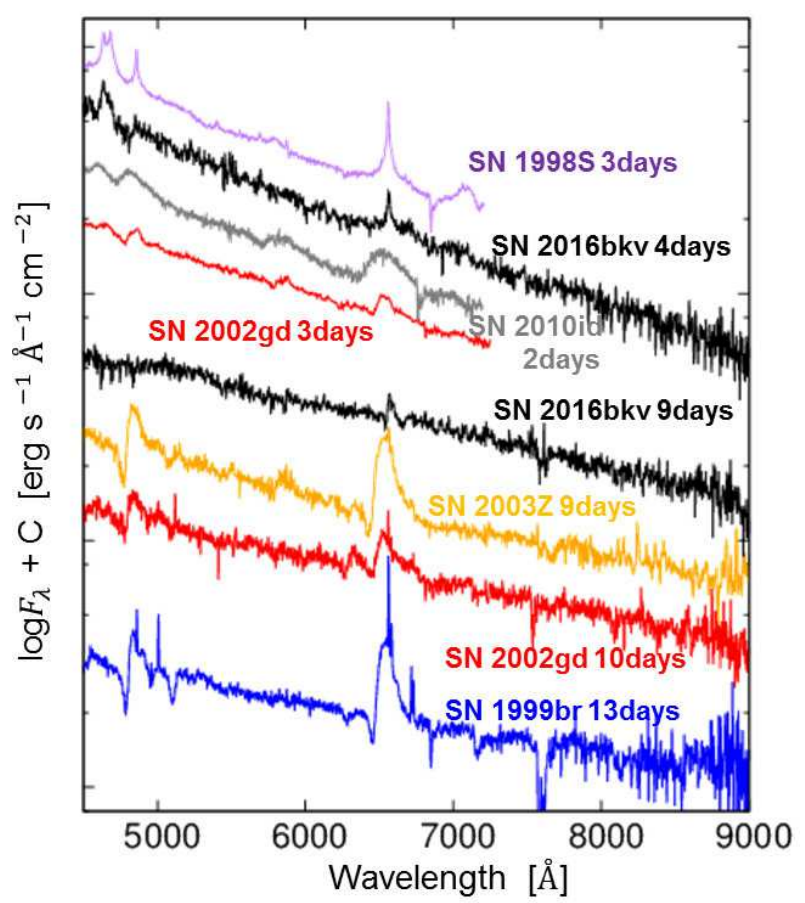

Figure 6. Spectra of SN 2016bkv in the early phase in comparison with LL SNe IIP 2002gd, 2003Z (Spiro et al.|2014), 1999br (Pastorello et al. 2004) and IIn 1998S (Fassia et al. 2001) at similar epochs. The fluxes are shown in the logarithmic scale and arbitrarily scaled to avoid overlaps.

\subsection{Spectral Features}

Figure 3 shows the spectral evolution of SN 2016bkv from 4 to 98 days. Until 20 days, the spectra are dominated by a blue continuum, superimposed by a weak and narrow ( $1000 \mathrm{~km} \mathrm{~s}^{-1}$ ) emission line of $\mathrm{H} \alpha$ (Figures 3 and 6). After 30 days after the explosion, various absorption lines, e.g., $\mathrm{H}$, 


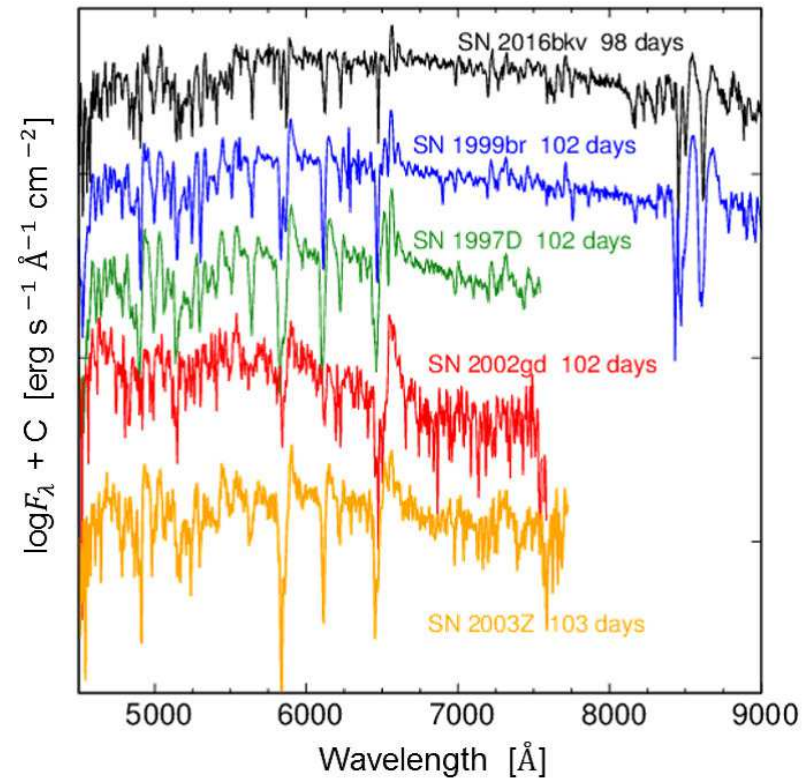

Figure 7. Spectra of SN 2016bkv at 98 days in comparison with the spectra of LL SNe IIP 1999br, 1997D (Pastorello et al. 2004), 2002gd and 2003Z (Spiro et al.2014) at similar epochs (near the end of the plateau phase).

Sc II, Fe II, Ba II, Ca II, get stronger with time (Figure 3). No significant $\mathrm{He}$ I line is seen in the spectra. In Figure 7 we compare the spectra of SN 2016bkv with those of LL SNe IIP at around 100 days. At this epoch, the overall spectral features are similar to those in other LL SNe IIP, although SN 2016bkv shows slightly narrower features than the others. At this epoch, SN 2016bkv shows a complicated structure of the $\mathrm{H} \alpha$ line, which is also visible in other LL SNe IIP. These facts confirm our classification of SN 2016bkv into LL SN IIP.

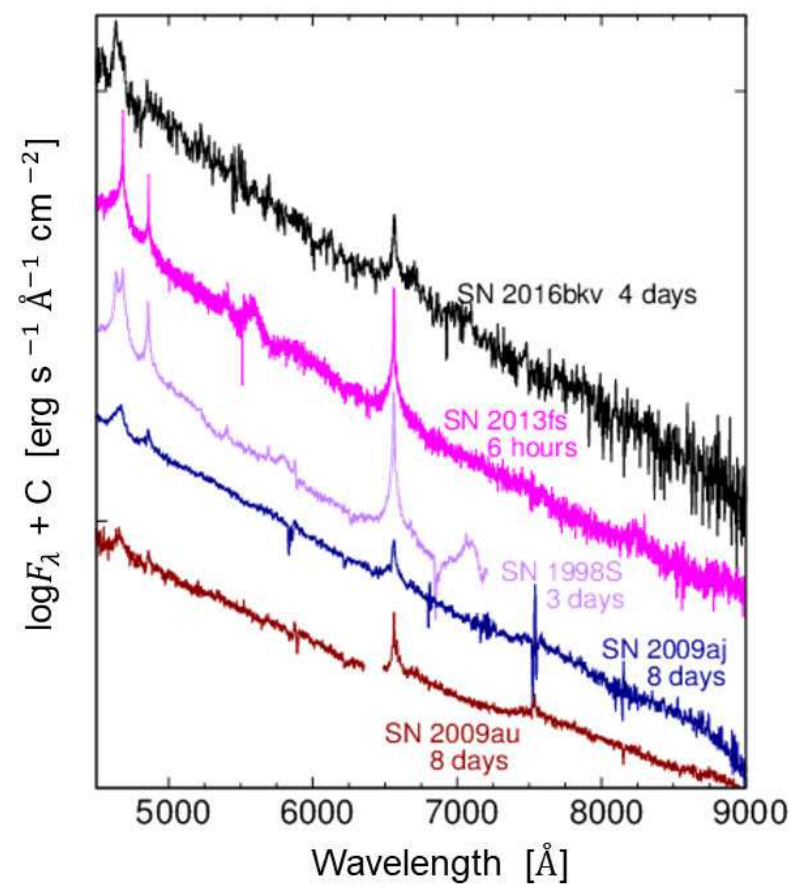

Figure 8. Spectrum of SN 2016bkv at 4 days in comparisons with those of SNe 1998S (Fassia et al. 2001), 2009aj, 2009au (Gutiérrez et al.2017) and 2013fs (Yaron et al. 2017) at their earliest phases (0.3-8 days).
Although the overall spectral properties of SN 2016bkv show similarities to other LL SNe IIP, SN 2016bkv shows some remarkable features in the earliest phases (Hosseinzadeh et al. 2018, ,see Figure 6). The $\mathrm{H} \alpha$ emission line at 4 days is narrower than in other LL SNe IIP, with a possible asymmetric profile truncated at a redder side, and there is no clear absorption component (see Figure 3). At 8-9 days, a possible weak absorption component in the bluer side of the $\mathrm{H} \alpha$ is marginally visible (The detail will be described in $\S 5.4$ ). After $\sim 9$ days, the absorption component gradually develops (Figure 3). All of these properties are quite different from those in well-observed LL SNe IIP 2002gd, 2003Z and 1999br (see Figure 6), which show clear P Cygni profiles in $\mathrm{H} \alpha$ (and $\mathrm{H} \beta$ ) at these epochs with larger line velocities.

Narrow Balmer emission lines with a blue continuum are sometimes seen in $\mathrm{SNe}$ II at their early phases. Figure 8 shows the spectral comparison of SN 2016bkv with other SNe II with narrow emission lines and a blue continuum at 0-8 days. In fact, the overall features of SN 2016bkv match well to those of SN IIn 1998S (Fassia et al. 2001), whose spectra show the emission lines of $\mathrm{H} \alpha$ and $\mathrm{H} \beta$, and also the emission lines around $4600 \AA$ (a blend of highly-excitation lines of C III, $\mathrm{N}$ III and He II ; Shivvers et al.2017). As also shown in Figure 8. similar emission features are seen in SNe 2009aj, 2009au at comparable epochs (Gutiérrez et al. 2017). The similarity is also found for SN 2013fs (iPTF13dqy; Yaron et al. 2017), as the spectrum was observed much earlier since the explosion. Given the epochs when these spectra are obtained, the emissions from SNe 2009aj and 2009au may be powered by the CSM interactions, while those of SN 2013fs are suggested to be flash ionized (FI) features (Gal-Yam et al. 2014). We discuss the origin of these features from a viewpoint of possible CSM-ejecta interaction in $\$ 5.3$ and $\$ 5.4$.

\subsection{Line Velocities}

After the end of the initial bump ( $\sim 30$ days after the explosion), many resolved absorption lines are seen in the spectra (Figure 3). We made line identifications by following Pastorello et al. (2004). In LL SNe IIP, the $\mathrm{H} \alpha$ absorption line around 50 days is often affected by Ba II $\lambda 6497$ because of the slight wavelength difference between $\mathrm{H} \alpha$ and Ba II $\lambda 6497$ (Roy et al. 2011; Lisakov et al. 2017). However, since the velocity of $\mathrm{H} \alpha$ of $\mathrm{SN} 2016 \mathrm{bkv}$ is slower than that of other LL SNe IIP (see Figure 9), the profile of $\mathrm{H} \alpha$ of SN 2016bkv can be clearly isolated from the Ba II $\lambda 6497$ between 45 and 98 days. The line velocities were measured by fitting a Gaussian function to each absorption line. Figure 9 shows the line velocity evolutions of various absorption lines: $\mathrm{H} \beta$, Fe II $\lambda 5169, \mathrm{Na}$ I D, Sc II $\lambda 6246, \mathrm{Ba}$ II $\lambda 6497, \mathrm{H} \alpha$ and $\mathrm{Ca}$ II $\lambda 8662$. If we compare the lines created by the same element at the same ionization state, the velocities and their evolutions are mutually consistent (e.g., Ca II NIR triplet). Thus only representative line velocities are shown in Figure 9 The velocities and their evolutions are consistent for each element; the velocities are about $1,600-2,000 \mathrm{~km} \mathrm{~s}^{-1}$ at 22 days and evolves to $1,000-1,600 \mathrm{~km} \mathrm{~s}^{-1}$ at 85 days. The Sc II and $\mathrm{Na}$ I $\mathrm{D}$ lines exhibit one of the lowest velocities among these lines.

In normal SNe IIP (e.g., SN 1999em), the H $\alpha$ line velocities are always higher than the velocities of other elements at all the epochs (Leonard et al. 2002a). This trend is, however, not necessarily true for LL SNe IIP. For example, the faster line velocity is observed for the $\mathrm{Ca}$ IR triplet and $\mathrm{Fe}$ II than the $\mathrm{H} \alpha$ in SN 2008in at 60-100 days after the explosion (Roy et al. 
2011). Similarly, in SN 2016bkv, the Ca IR triplet line velocities are higher than the $\mathrm{H} \alpha$ line velocity after $\sim 80$ days.

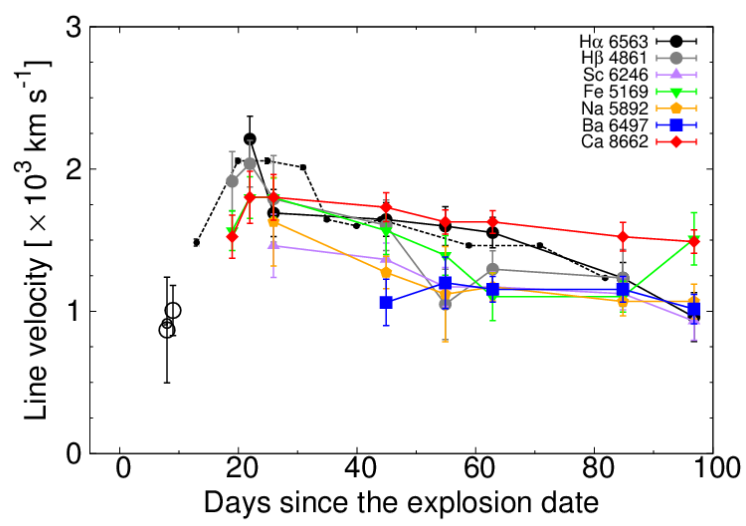

Figure 9. Velocity evolutions of the $\mathrm{H} \alpha, \mathrm{Sc}$ II, Fe II, Na I D, and $\mathrm{Ca}$ II lines in SN 2016bkv. The open black circles show the line velocity of the weak absorption component accompanied with the narrow $\mathrm{H} \alpha$ emission line seen at 8 and 9 days (see $\S 4.2$ ). We obtain the errors by taking into account the uncertainties in the wavelength calibration and fitting error. In addition to the measurements for our spectra, we also added $\mathrm{H} \alpha$ line velocities measured in the spectra presented by Hosseinzadeh et al. (2018, dashed line).

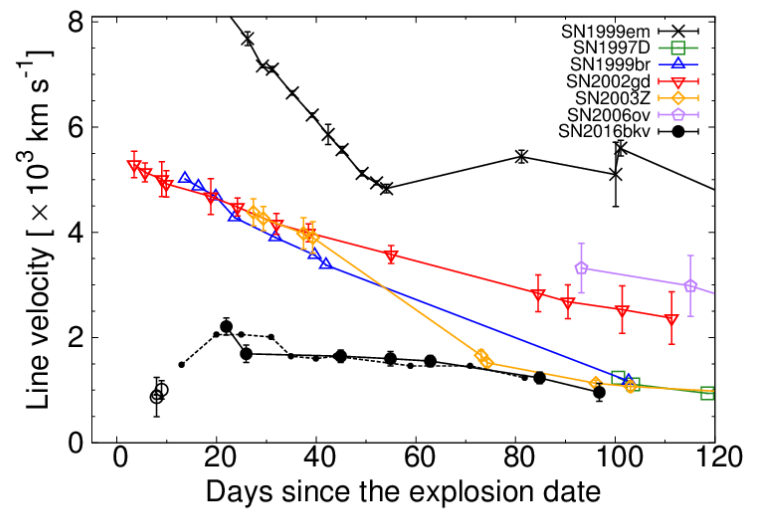

Figure 10. Comparison of $\mathrm{H} \alpha$ line velocities among LL IIP SNe 2016bkv, 1997D and 1999br (Pastorello et al. 2004), 2002gd, 2003Z, 2006ov (Spiro et al. 2014), and a normal SN IIP 1999em (Leonard et al. 2002a). The dashed line for SN 2016bkv shows the velocities measured in the spectra by Hosseinzadeh et al. (2018).

Figure 10 shows the $\mathrm{H} \alpha$ velocity evolution of SN 2016bkv, compared with other LL SNe and the normal SN IIP 1999em (Leonard et al. 2002a). It is clear that LL SNe IIP commonly show lower velocities than those of SN IIP 1999em over the entire phases. SN 2016bkv exhibits the lowest velocities among LL SNe IIP until $\sim 60$ days. Interestingly, SN 2016bkv shows a slower evolution; the evolution of line velocities after $\sim 20$ days is flatter than the other LL SNe IIP. The slow recession of the photospheric velocity means that the photospheric radius $\left(R_{\mathrm{ph}}\right)$ tends to be large. This may be related to the slight increase in the luminosity at the end of the plateau phase $\left(L \propto R_{\mathrm{ph}}^{2} T^{4}\right)$ seen in SN 2016bkv.

The $\mathrm{H} \alpha$ velocities at the earliest phases are of interest. At 4,5 , and 7 days, the spectra exhibit the emission line of $\mathrm{H} \alpha$
, and then, a very weak absorption component is possibly detected at 8 and 9 days. The velocities of these absorption components are measured as $800-1,000 \mathrm{~km} \mathrm{~s}^{-1}$ (open symbols in Figures 9 and 10). In these figures, we also added the measured velocities using the spectra presented by Hosseinzadeh et al. (2018, dashed line). In addition to the absorption lines, we also measure the width of the emission line of $\mathrm{H} \alpha$. We find that the FWHMs of the emissions are similar to the blueshift velocities of the absorption lines, i.e., 800 $1,000 \mathrm{~km} \mathrm{~s}^{-1}$. These velocities are clearly slower than the velocities of the stronger absorption component which appears after 26 days $\left(1,700 \mathrm{~km} \mathrm{~s}^{-1}\right)$. Implication from this feature is discussed in $\$ 5.4$

\section{DISCUSSION}

\subsection{Bolometric Luminosity and ${ }^{56} \mathrm{Ni}$ Mass}

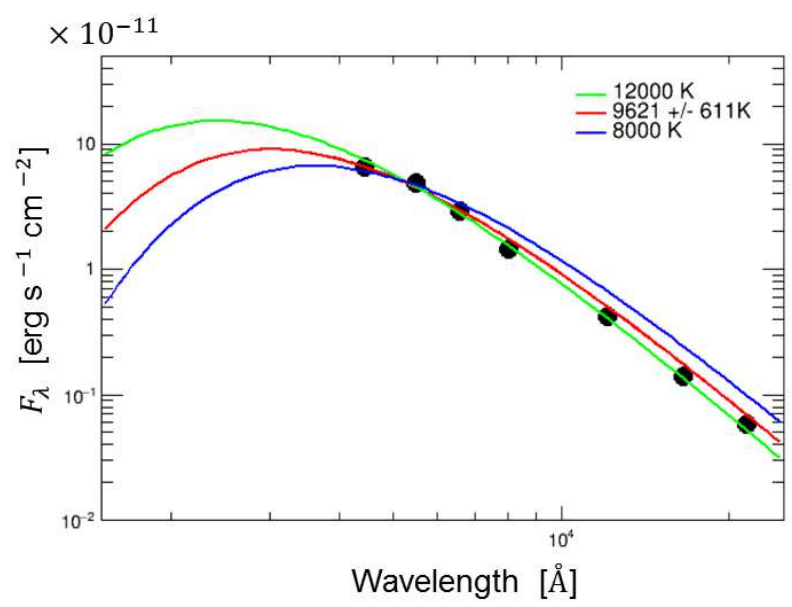

Figure 11. SED of SN 2016bkv at 7.4 days after the explosion as compared to blackbody with different temperatures: the best fit model (in red), models of higher temperature $(8000 \mathrm{~K}$, in blue) and lower one (12000K, in green).

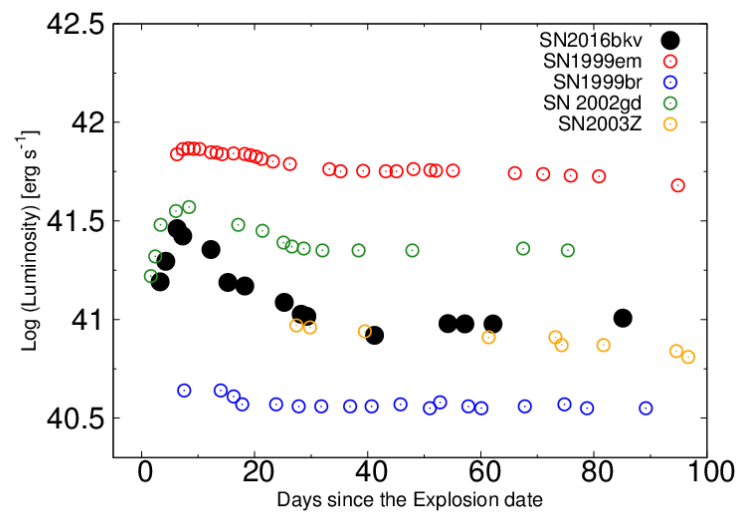

Figure 12. Quasi-bolometric light curve of SN 2016bkv compared with that of LL SNe IIP 1999br (Pastorello et al. 2004), 2002gd, 2003Z (Spiro et al. 2014) and a normal SN IIP 1999em (Leonard et al. 2002a). For all the $\mathrm{SNe}$, the quasi-bolometric luminosities are derived by blackbody fitting in the $B V R I$-band wavelength range.

We derived quasi-bolometric luminosities by integrating the $B V R I$-band fluxes as follows. First, the extinction-corrected 
$B V R I$-band magnitudes were converted into fluxes using filter parameters (Bessell 1990) and the zero-magnitude fluxes (Fukugita et al. 1995). Then, we fitted the spectral energy distributions (SEDs) with the blackbody function. Finally the flux is integrated by using the best-fit blackbody parameters. In the blackbody fit, we have included the $J H K_{s}$-band if available, in order to constrain the temperature as accurately as possible. We then integrated fluxes in the BVRI-band wavelength region. The quasi-bolometric luminosities of other LL SNe IIP were also calculated in the same manner. This allows fair comparisons to the other samples, as the NIR data are not always available.

Figure 11 shows the SED with $B V R I J H K_{s}$-band flux and the best-fit blackbody function at 7.4 days after the explosion (at the peak of the early bump). The blackbody temperature is obtained to be $9,600 \pm 600 \mathrm{~K}$. The temperature of LL SNe IIP $2002 \mathrm{gd}$ at 5.7 and 9.1 days are $12,700 \mathrm{~K}$ and 9,700 K, respectively (Spiro et al. 2014). Note that since there is no data at ultraviolet wavelengths and the temperature estimate in particular at $T>10,000 \mathrm{~K}$ is somewhat uncertain. To illustrate this uncertainty, the blackbody functions with the temperature of $\sim 12,000$ and $\sim 8,000 \mathrm{~K}$ are also shown in Figure 11. This uncertainty affects the bolometric luminosities up to by a factor of about two.

Figure 12 shows the quasi-bolometric light curve of SN 2016bkv, compared with those of other LL SNe IIP and the normal SN IIP 1999em. As in the multi-color light curves, the bolometric luminosity of SN 2016bkv clearly shows a bump ( $\gtrsim 1 \mathrm{mag}$ ) in the early phase. As expected, the luminosities of SN 2016bkv are always $\sim 7-10$ times fainter than those of the normal SN IIP 1999em. In comparisons with LL SNe IIP, the luminosities of SN 2016bkv are comparable to those of SN 2002gd before peak. After the bump ( $\sim 30$ days), the luminosities of SN 2016bkv become 2-3 times fainter than those of SN 2002gd, and become comparable to those of SN $2003 Z$.

Finally we give a constraint on the ${ }^{56} \mathrm{Ni}$ mass of SN 2016bkv using the luminosity at the tail. As discussed in $\S 2$, we only obtain an upper limit of the luminosity in the late phase due to the background emission from the host galaxy. By using the quasi-bolometric luminosity at 201 day and assuming a relation between the tail luminosity and the ${ }^{56} \mathrm{Ni}$ mass suggested by Hamuy (2003), we obtain the upper limit of $M\left({ }^{56} \mathrm{Ni}\right) \lesssim 0.01 M_{\odot}$. Our upper limit of the optical luminosity at $\sim 200$ days is close to the observed luminosity presented by Hosseinzadeh et al. (2018). Therefore, the ejected mass of ${ }^{56} \mathrm{Ni}$ is, in fact, likely to be $M\left({ }^{56} \mathrm{Ni}\right) \sim 0.01 M_{\odot}$. This is between the typical ${ }^{56} \mathrm{Ni}$ masses of other LL SNe IIP and those of normal SNe IIP (Spiro et al. 2014).

\subsection{Progenitor}

The observed properties of SNe IIP reflect the radius and mass of the progenitor and the energy of the explosion. If the density structure and temperature are described in a selfsimilar manner for different $\mathrm{SNe}$, the timescale of the plateau $\left(t_{\mathrm{EoP}, \mathrm{sn}} \sqrt{16}\right.$ and the plateau luminosity $\left(L_{s n}\right)$ are scaled as follows (Kasen \& Woosley 2009):

$$
t_{\mathrm{EoP}, \mathrm{sn}} \propto E^{-1 / 6} M_{\mathrm{ej}}^{1 / 2} R_{0}^{1 / 6} \kappa^{1 / 6} T_{I}^{-2 / 3}, \text { and }
$$

\footnotetext{
${ }^{16}$ In this context, we consider that the bump component is additional to the underlying plateau one. Thus, we define $t_{\mathrm{EoP}, \mathrm{sn}}$ as the epoch of the end of the plateau.
}

$$
L_{\mathrm{SN}} \propto E^{5 / 6} M_{\mathrm{ej}}^{-1 / 2} R_{0}^{2 / 3} \kappa^{-1 / 3} T_{I}^{4 / 3},
$$

where $E$ is the kinetic energy of the ejecta, $M_{\mathrm{ej}}$ is the ejecta mass, $R_{0}$ is the radius of progenitor star, $\kappa$ is the opacity, and $T_{I}$ is the ionization temperature. Replacing the kinetic energy by the ejecta velocity $\left(v_{\mathrm{ej}}\right)$, using $E \propto M_{\mathrm{ej}} v_{\mathrm{ej}}^{2}$, the relations (1) lead to the following expressions:

$$
\begin{aligned}
t_{\mathrm{EoP}, \mathrm{sn}} & \propto v_{\mathrm{ej}}^{-1 / 3} M_{\mathrm{ej}}^{1 / 3} R_{0}^{1 / 6} \kappa^{1 / 6} T_{I}^{-2 / 3}, \text { and } \\
L_{\mathrm{SN}} & \propto v_{\mathrm{ej}}^{5 / 3} M_{\mathrm{ej}}^{1 / 3} R_{0}^{2 / 3} \kappa^{-1 / 3} T_{I}^{4 / 3} .
\end{aligned}
$$

Since it is a sound approximation that $\kappa$ and $T_{I}$ are the same for different $\mathrm{SNe}$, two quantities, $M_{\mathrm{ej}}$ and $R_{0}$, are constrained from $t_{\mathrm{EoP}, \mathrm{sn}}, L_{\mathrm{SN}}$, and $v_{\mathrm{ej}}$.

Here we estimate the properties of SN 2016bkv, using equations above. We first use SN $2003 \mathrm{Z}$ for reference, whose properties have been extensively studied with numerical light-curve models (Pumo et al. 2017, $M_{03 Z}=$ $\left.11.3 M_{\odot}, R_{03 Z}=260 R_{\odot}\right)$. As the photospheric velocity, we use the line velocity of the Sc II line (Leonard et al. 2002a). The ratio of velocity of $\mathrm{SN} 2016 \mathrm{bkv}$ to that of LL SN IIP $2003 Z\left(v_{16 \mathrm{bkv}} / v_{03 Z}\right)$ is $\sim 0.8$ around 60 days. Using this velocity ratio, the ratio of $t_{\mathrm{EoP}, \mathrm{sn}}$ between SN 2016bkv $\left(t_{16 \mathrm{bkv}}\right)$ and SN 2003Z $\left(t_{03 \mathrm{Z}}\right)$ is expressed as follows: $t_{16 \mathrm{bkv}} / t_{03 \mathrm{Z}} \sim 1.1\left(M_{16 \mathrm{bkv}} / M_{03 \mathrm{Z}}\right)^{1 / 3}\left(R_{16 \mathrm{bkv}} / R_{03 \mathrm{Z}}\right)^{1 / 6}$. We can similarly derive the ratio of the luminosity as $\left(L_{16 \mathrm{bkv}} / L_{03 \mathrm{Z}}\right) \sim 0.65\left(M_{16 \mathrm{bkv}} / M_{03 \mathrm{Z}}\right)^{1 / 3}\left(R_{16 \mathrm{bkv}} / R_{03 \mathrm{Z}}\right)^{2 / 3}$. From our data, $t_{16 \mathrm{bkv}} / t_{03 \mathrm{Z}}$ is given as $\sim 1.4$ (see Figure 4 ) and $L_{16 \mathrm{bkv}} / L_{03 Z}$ is $\sim 1.0$ (see Figure 12 ). Here we use $L_{03 Z}=$ $1 \times 10^{41} \mathrm{erg} \mathrm{s}^{-1}$ and $t_{03 \mathrm{Z}}=110$ days, respectively. Therefore, we derive $M_{16 \mathrm{bkv}} / M_{03 \mathrm{Z}} \sim 1.7$ and $R_{16 \mathrm{bkv}} / R_{03 \mathrm{Z}} \sim 1.5$, suggesting that the ejecta mass is larger in SN 2016bkv.

We also perform similar analysis using SNe 1999br and 2008bk (Pastorello et al. 2004; Lisakov et al. 2017). We obtain similar tendencies, i.e., a larger ejecta mass than these LL SNe IIP, i.e., 1.2 and 2.4 times larger than these objects, respectively. By adopting the explosion parameters presented by Zampieri et al. (2003); Lisakov et al. (2017); Pumo et al. (2017), we derive the ejecta mass and progenitor radius of SN 2016bkv to be $16.1-19.4 M_{\odot}$ and $180-1080 R_{\odot}$, respectively (Spiro et al. 2014). It should be noted, however, that since the methods to estimate the explosion parameters are different in different papers, the absolute values include large systematic uncertainties.

From these results, we suggest that the progenitor of SN 2016bkv is more massive than the progenitors of other LL SNe IIP. It should be noted that Hosseinzadeh et al. (2018) suggest a relatively low mass $\left(\sim 9 M_{\odot}\right)$ progenitor from the nebular spectra, in particular from the weakness of the [O I] line. However, such a low-mass progenitor is unlikely to result in a late $t_{\text {EoP.sn }}$ for $\gtrsim 140$ days as derived for SN $2016 \mathrm{bkv}$ (see Sukhbold et al. 2016). On the other hand, our estimate using the properties observed of the plateau phase has a different possible systematic error, based on the assumption that the same scaling relations of normal SNe IIP (Eqs. 1 and 2) are applicable to LL SNe IIP. Therefore, the derived properties are subject to uncertainties. We need more detailed, consistent modeling of both light curves and spectra from the early to nebular phases.

\subsection{Properties of CSM Inferred from the Early Bump Emission}


SN 2016bkv shows a clear bump in the optical light curves in the early phases. In addition, the early spectra show a narrow $\mathrm{H} \alpha$ emission line with a blue continuum. These properties suggest the interaction between the SN ejecta and the circumsetellar matter (CSM) as in SNe IIn. The signatures of the interaction are seen until $\sim 20$ days.

The luminosity arising from the interaction between the SN ejecta and CSM can be estimated as

$$
L=4 \pi r_{s}^{2} \cdot \frac{1}{2} \rho v_{\mathrm{ej}}^{3} \epsilon
$$

where $r_{s}$ is the radius of the shock and $\epsilon \sim 0.1$ is a conversion factor from the kinetic energy to radiation (Moriya \& Maeda 2014). At the peak of the bump, 7.4 days after the explosion, the luminosity of the bump component is $L=4 \times 10^{41} \mathrm{erg} \mathrm{s}^{-1}$ . Here we extract the luminosity of the bump component from the total luminosity at 7-9 days by subtracting the plateau luminosity at 40-60 days (assuming that the SN luminosity is constant). The plateau component accounts for about one third of the total flux (see Figure 12). Assuming that the ejecta velocity is $v_{\mathrm{ej}} \sim 2,000 \mathrm{~km} \mathrm{~s}^{-1}$, which is observed in $\mathrm{H} \alpha$ absorption at 22 days (Figure 9), the radius reaches $r_{s} \sim 1,800 R_{\odot}$ at the peak of the bump. Then we obtain the mass loss rate of $\dot{M}_{0} / u_{v, 5}=1.7 \times 10^{-3} M_{\odot} \mathrm{yr}^{-1}$ where $\dot{M}_{0}$ is the mass loss rate and $u_{v, 5}$ is the wind velocity normalized to $10^{5} \mathrm{~cm} \mathrm{~s}^{-1}$. The CSM mass within $r_{s}$ is $M_{\mathrm{CSM}} \sim 6.8 \times 10^{-2} M_{\odot}$.

If we assume a typical velocity of the red supergiant (RSG) wind, $u_{v, 5}=10$ (i.e., $10 \mathrm{~km} \mathrm{~s}^{-1}$ ), the mass loss rate is $\dot{M} \sim$ $1.7 \times 10^{-2} M_{\odot} \mathrm{yr}^{-1}$. Then, the CSM within the shock radius $r_{s}$ corresponds to the mass loss in the final 4.1 years. These results are consistent with those presented by other studies for the SNe II showing early bumps (e.g., Morozova et al. 2017; Moriya et al. 2017), suggesting that the progenitor of SN 2016bkv has also experienced an intensive mass loss just before the $\mathrm{SN}$ explosion.

The dense CSM derived above might also follow a strong flash ionized (FI) features (e.g., Gal-Yam et al. 2014; Khazov et al. 2016), if there would have been a sufficiently strong ionizing flux 17 . However, at the peak of the bump, this effect is likely insignificant already, since the FI features are typically found only within the first day as seen in SN 2013cu and some other SNe (Gal-Yam et al. 2014) and soon disappear following the decay of the UV flux from an SN. In addition, SN 2016bkv is a LL SN IIP and the ionizing photon would be less intense than those in normal SNe IIP, leading to fainter FI features. Therefore, we do not attribute the emission lines in the early phase to the FI features. It is possible that SN 2016bkv might have shown a genuine FI feature, if it would have been observed within the first day. Adopting the properties of CSM as derived above, we estimate that a strong $\mathrm{H} \alpha$ emission $\left(L_{H \alpha} \sim 7 \times 10^{40} \mathrm{erg} \mathrm{s}^{-1}\right)$ should have existed if such an observation would have been performed within a day after the explosion. Interestingly, SN 2013cu and SN 2016bkv show common emission lines of $\mathrm{C}$ III/ $\mathrm{N}$ III at $\sim 3-4$ days. These features may be caused by ionizing photons from the on-going interacting region.

\subsection{Possible Eruptive Mass Loss}

\footnotetext{
${ }^{17}$ We use the terminology of FI features for the features produced by radiative ionization from shock breakout either at the stellar surface or at the dense CSM.
}

During several days just after the bump peak (7-10 days after the explosion), spectra of SN 2016bkv show a hint of a weak $\mathrm{H} \alpha$ absorption with the velocities of $800-1000 \mathrm{~km} \mathrm{~s}^{-1}$. The origin of this feature could be distinct from the strong $\mathrm{P}$ Cygni features with the broader emission lines after $\sim 26$ days (Figure 3), which are produced by the SN ejecta. As discussed in $\S 5.3$, the bump in the light curve and narrow emission lines at the early phases are likely to be caused by the CSM interaction. In this context, we speculate that the absorption component might be originated in the unshocked CSM. The observed velocities (800-1,000 $\mathrm{km} \mathrm{s}^{-1}$ ) are much higher than a typical wind velocity of RSGs. This suggests that the progenitor star might have experienced a sudden, eruptive mass loss ejecting $\sim 6.8 \times 10^{-2} M_{\odot}$ within $r_{s}$ at only 0.2 years prior to the explosion. Although it is rather speculative, it may be the case given the large uncertainty in the mass loss of the progenitor just before the explosion. However, the gradual increase of the $\mathrm{H} \alpha$ velocities from 1,000 to $2000 \mathrm{~km} \mathrm{~s}^{-1}$ (Figure 9], is not well explained if the low velocity component is attributed to unshocked CSM. Dense observations at the early phases are necessary to fully understand possible activities of the progenitor of LL SNe IIP.

\section{CONCLUSIONS}

We present our optical and NIR observations of the lowluminosity Type IIP SN 2016bkv. Our observations cover the initial rising phase through the plateau phase, and make this object one of the best observed LL SNe IIP. The end of the plateau is the latest ( $\gtrsim 140$ days) among other LL SNe IIP. In the initial phase, SN 2016bkv clearly shows a bump ( $\gtrsim 1 \mathrm{mag}$ ) in the light curves. The bump peaks at $\sim 7$ days after the explosion. During this initial phase, optical spectra show a blue continuum and narrow $\mathrm{H}$ emission lines. These features suggest the interaction between the SN ejecta and the CSM.

Using the scaling relations for the timescale of the plateau and its luminosity, the ejecta mass of SN 2016bkv is estimated to be larger than those of other LL SNe IIP. From the luminosity of the early bump and the $\mathrm{H} \alpha$ line emission, we conclude that the progenitor star has a relatively massive CSM in the vicinity of the progenitor star. If the CSM has been produced by the stellar wind in the progenitor, the required mass loss rate is estimated to be $\sim 1.7 \times 10^{-2} M_{\odot} \mathrm{yr}^{-1}$ and the accumulated mass of the CSM is $\sim 6.8 \times 10^{-2} M_{\odot}$ at least within several years before the SN explosion (for the assumed wind velocity of $10 \mathrm{~km} \mathrm{~s}^{-1}$ ). The mass loss rate inferred for SN 2016bkv is comparable to or even larger than the largest mass loss rate estimated for RSG stars in the Milky Way (e.g., $\sim 10^{-3} M_{\odot} \mathrm{yr}^{-1}$ for VY CMa; Smith et al. 2009).

This research has made use of the NASA/IPAC Extragalactic Database (NED) which is operated by the Jet Propulsion Laboratory, California Institute of Technology, under contract with the National Aeronautics and Space Administration.

This research has made use of the NASA/ IPAC Infrared Science Archive, which is operated by the Jet Propulsion Laboratory, California Institute of Technology, under contract with the National Aeronautics and Space Administration.

KM acknowledges support provided by Japan Society for the Promotion of Science (JSPS) through KAKENHI Grant $17 \mathrm{H} 02864$.

\section{REFERENCES}


Akitaya, H., et al. 2014, in Proc. SPIE, Vol. 9147, Ground-based and Airborne Instrumentation for Astronomy V, 914740

Anderson, J. P., et al. 2014, ApJ, 786, 67

Bessell, M. S. 1990, PASP, 102, 118

Bose, S., et al. 2015, ApJ, 806, 160

Chugai, N. N., \& Utrobin, V. P. 2000, A\&A, 354, 557

Dessart, L., John Hillier, D., \& Audit, E. 2017, A\&A, 605, A83

Faran, T., et al. 2014, MNRAS, 442, 844

Fassia, A., et al. 2001, MNRAS, 325, 907

Filippenko, A. V. 1997, ARA\&A, 35, 309

Fraser, M., et al. 2011, MNRAS, 417, 1417

Fukugita, M., Shimasaku, K., \& Ichikawa, T. 1995, PASP, 107, 945

Gal-Yam, A., et al. 2014, Nature, 509, 471

Galbany, L., et al. 2016, AJ, 151, 33

Graur, O., Bianco, F. B., Modjaz, M., Shivvers, I., Filippenko, A. V., Li, W., \& Smith, N. 2017, ApJ, 837, 121

Gutiérrez, C. P., et al. 2017, ApJ, 850, 89

Hamuy, M. 2003, ApJ, 582, 905

Hosseinzadeh, G., Howell, D. A., Arcavi, I., Mccully, C., \& Valenti, S. 2016, Transient Name Server Classification Report, 239

Hosseinzadeh, G., et al. 2018, ArXiv e-prints

Itagaki, K. 2016, Transient Name Server Discovery Report, 234

Kasen, D., \& Woosley, S. E. 2009, ApJ, 703, 2205

Kawabata, K. S., et al. 2008, in Proc. SPIE, Vol. 7014, Ground-based and Airborne Instrumentation for Astronomy II, 70144L

Khazov, D., et al. 2016, ApJ, 818, 3

Kitaura, F. S., Janka, H.-T., \& Hillebrandt, W. 2006, A\&A, 450, 345

Leonard, D. C., et al. 2002a, PASP, 114, 35

-. 2002b, AJ, 124, 2490

Li, W., et al. 2011, MNRAS, 412, 1441

Lisakov, S. M., Dessart, L., Hillier, D. J., Waldman, R., \& Livne, E. 2017, MNRAS, 466, 34

-. 2018, MNRAS, 473, 3863

Moriya, T. J., \& Maeda, K. 2014, ApJ, 790, L16
Moriya, T. J., Yoon, S.-C., Gräfener, G., \& Blinnikov, S. I. 2017, MNRAS, 469, L108

Morozova, V., Piro, A. L., \& Valenti, S. 2017, ApJ, 838, 28

Pastorello, A., et al. 2009, MNRAS, 394, 2266

-. 2004, MNRAS, 347, 74

Pejcha, O., \& Prieto, J. L. 2015, ApJ, 806, 225

Persson, S. E., Murphy, D. C., Krzeminski, W., Roth, M., \& Rieke, M. J. 1998, AJ, 116, 2475

Poznanski, D., Prochaska, J. X., \& Bloom, J. S. 2012, MNRAS, 426, 1465

Pumo, M. L., Zampieri, L., Spiro, S., Pastorello, A., Benetti, S., Cappellaro, E., Manicò, G., \& Turatto, M. 2017, MNRAS, 464, 3013

Roy, R., et al. 2011, ApJ, 736, 76

Sanders, N. E., et al. 2015, ApJ, 799, 208

Schlafly, E. F., \& Finkbeiner, D. P. 2011, ApJ, 737, 103

Shivvers, I., et al. 2017, PASP, 129, 054201

Smith, N., Hinkle, K. H., \& Ryde, N. 2009, AJ, 137, 3558

Spiro, S., et al. 2014, MNRAS, 439, 2873

Stetson, P. B. 1987, PASP, 99, 191

Sukhbold, T., Ertl, T., Woosley, S. E., Brown, J. M., \& Janka, H.-T. 2016, ApJ, 821, 38

Takáts, K., et al. 2015, MNRAS, 450, 3137

Tody, D. 1986, in Proc. SPIE, Vol. 627, Instrumentation in astronomy VI, ed. D. L. Crawford, 733

Tody, D. 1993, in Astronomical Society of the Pacific Conference Series, Vol. 52, Astronomical Data Analysis Software and Systems II, ed. R. J. Hanisch, R. J. V. Brissenden, \& J. Barnes, 173

Turatto, M., et al. 1998, ApJ, 498, L129

Valenti, S., et al. 2016, MNRAS, 459, 3939

Yaron, O., et al. 2017, Nature Physics, 13, 510

Zampieri, L., Pastorello, A., Turatto, M., Cappellaro, E., Benetti, S., Altavilla, G., Mazzali, P., \& Hamuy, M. 2003, MNRAS, 338, 711 